\title{
Vitamin B blood plasma deficiency model for the study of diabetes complications demonstrates potential for the cure and prevention of complications in type 2 diabetes mellitus patients
}

\begin{abstract}
Introduction: The glucose model for the study of diabetes complications is too general to be useful in finding cure for, or prevention of, diabetes complications. A model, based on blood plasma Vitamin B Deficiency in diabetes mellitus patients, explains why diabetes complications selectively affect some organs and resist traditional treatment. It predicts that the daily blood plasma vitamin B deficiency initiates a concatenation of events that culminate in organ dysfunction described as diabetes complications and that complications affecting all organs are curable and preventable with vitamin B supplementation. This was tested.
\end{abstract}

Method: A continuous Open Trial was started in 2008 with one a day ingestible tablets given to type 2 diabetes mellitus patients with symptoms of diabetes complications affecting one or more organs. The tablet contained nicotinamide $(15.0 \mathrm{mg})$, thiamine hydrochloride $(12.5 \mathrm{mg})$ and calcium-d-pentothenate $(11.0 \mathrm{mg})$.

Results: Cures of early-stage diabetes complications affecting the eye, kidney, and the skin were reported. Continued use of the therapy has prevented reappearance of symptoms of diabetes complications for over 6years. No adverse effect has been reported.

Conclusion: The cures, improvements and prevention of diabetes complications accomplished by the therapy predicted by the Blood Plasma Vitamin B Deficiency model makes the model a potent tool in the study of diabetes complications.

Perspective: Future studies involving more patients, including insulin dependants, will be useful. Preventing diabetes complications with vitamin B supplementation as prophylactic represents a major breakthrough, in both human and economic terms, in the fight against the predicted type 2 diabetes mellitus epidemic.

Keywords: diabetes complications cured, chronic plasma vitamin b deficiency, malfunctioning microcirculation, tissue damage, therapy for cure and prevention
Volume 2 Issue 2 - 2015

\author{
Bempah Owusu Akyiaw \\ United Kingdom
}

Correspondence: Bempah Owusu Akyiaw, 362, Summerwood Road, Isleworth, Middlesex, TW7 7QP, United Kingdom, Tel 020 8892 II23,Email fenomin@hotmail.com

Received: February 03, 2015 | Published: April 07, 2015
Abbreviations: AGES, advanced glycation end products; $\mathrm{VbD}$, vitamin b deficiency; b3, niacin: b1, thiamine; b5, calcium-dpantothenate; LDVbT, low dose vitamin b therapy; HDB/T, high dose benfotiamine/thiamine therapy; TPP, thiamine pyrophosphate

\section{Introduction}

The glucose model for studying diabetes complications has produced two main theories: intracellular sorbitol accumulation theory to explain cataract formation in the eye and glycation theory which posits that the reaction products resulting from glucose proteins interactions advanced glycation end products, (AGES)cause diabetes complications. None of these theories proposes a therapy for the cure or prevention of diabetes complications. The glycation theory does not explain why some organs and not others develop diabetes complications. Besides, there seems to be no link between manifestation of complications in one organ and another, thus making it necessary to find separate cure for each affected organ. In spite of this, Babani \& Thornalley ${ }^{1}$ have expressed the view that, "the therapeutic approaches for further improvement in risk reduction and reversal of early stage nephropathy are likely to be effective if they act downstream of blood glucose control improving one or more pathways of biochemical dysfunction activated in hyperglycemia". This path is a cul-de-sac. A new model, based on blood plasma Vitamin B Deficiency (VbD) is proposed.

\section{Background}

Thornalley et al. ${ }^{2}$ found that at the end of the 24hour day, volunteers without diabetes mellitus retained, on average, $64.1 \mathrm{nmol} / 1$ of the water-soluble thiamine (vitamin b1) in the blood plasma; the type 1 diabetes mellitus patients, $15.3 \mathrm{nmol} / 1$; and the type 2 patients, $16.3 \mathrm{nmol} / 1$. The loss of vitaminb1 through urinary excretion was fourfold in type 1 and three-fold in type 2 patients.

\section{The model}

If these ratios of blood plasma vitamin b1 retention obtained in the experimental setting are applied to daily, diet--sourced, water-- 
soluble vitamin B intake, the following scenario emerges. Sometime after breakfast, both the volunteers and persons with diabetes mellitus receive adequate supply of vitamin $\mathrm{B}$, [including niacin (b3) and thiamine (b1), the primary and secondary vasodilators respectively, that sustain the integrity of the microcirculation], in the blood plasma. Full vasodilatation in the microcirculation in the organs of the volunteers as well as persons with diabetes mellitus will be sustained and normal exchange of gases, nutrients and particles between blood and tissues would occur. Also, all the subjects will start losing vitamin B through urinary excretion, but the loss will be made up by subsequent meals throughout the day. However, between the last meal and breakfast the following day, (fasting period), there is loss of vitamin B without replenishment.

From the experimental blood plasma vitamin b1 retention ratios, it is inferred that the concentration of blood plasma vitamin in the volunteers will be sufficient to sustain full vasodilatation in the microcirculation in organs during the entire fasting period. Exchange of gases, nutrients and particles between blood and tissues will not be affected in persons in this group. With the diabetes mellitus patients, however, at some point during this period especially, in the early morning hours before breakfast, the blood plasma concentrations of niacin (b3) and thiamine (b1) fall below the minimum required to sustain full vasodilatation in the microcirculation. Diminished vasodilatation in the microcirculation occurs and persists till after breakfast when vitamin B is replenished again.

During the period of diminished vasodilatation, the microcirculation malfunctions adversely impacting on the exchange of gases, nutrients and particles between blood and tissues. This causes tissue damage. The cycle is repeated everyday in the life of the diabetes mellitus patient.

In the organs with microcirculation such as, the brain, eye, heart, kidney and the skin, the daily tissue damage accumulates over time and manifests as organ dysfunction. There are early warning signs of organ deterioration in the eye, (retinopathy); the kidney (swollen feet); and the skin (sores); but the brain and the heart do not give any signs before symptoms of organ dysfunction manifest as stroke and heart attack. The early symptoms of organ deterioration and dysfunction such as, blindness, heart and kidney failures and stroke, are collectively described as diabetes complications.

\section{Conclusions arising from the model}

The model demonstrates that the development of diabetes complications is independent of blood plasma glucose-hyperglycemia. Therefore, the conclusions deduced from this model apply equally to people with all types of diabetes mellitus, and even, to those who are not, or cannot be, clinically diagnosed as diabetes mellitus patients but can, nevertheless, develop symptoms similar to diabetes complications. The model predicts that only organs with microcirculation manifest diabetes complications. The brain, eye, heart, kidney, and the skin are the organs known to develop diabetes complications. The lungs, the liver, the spleen, the connective tissues, etc; do not.

Diabetes complications develop simultaneously in each of the susceptible organs as soon as a person develops diabetes mellitus, even before diagnosis. Some organs show early warning symptoms of deteriorating organ function, but others do not. They all may become dysfunctional, eventually. Tissue damage occurs daily, therefore, with time, symptoms manifest and exacerbate in intensity in one or more organs. For example, in the eye, vision diminishes progressively with time. During this period, the skin may develop sore; the risk of heart attack, stroke and kidney failures also increases.

Finally, if the chain of events leading to organ deterioration and dysfunction can be permanently broken or disrupted, prevention or cure of diabetes complications can be achieved. This offers the opportunity to develop a therapy that cures or prevents diabetes complications. Such a therapy will stop tissue damage simultaneously in all organs for natural healing to take place. Therefore, a therapy that cures one manifested symptom of diabetes complications in any one organ, will simultaneously cure, or prevent the development of, symptoms in all organs; obviating the need to find separate drugs to treat individual deteriorating organ. The converse is also true: withdrawing treatment will affect all organs and cause them to revert to the "status quo ante", that is, the cure or improvement will be lost with time.

\section{Hypothesis}

To validate the conclusions deduced from the model, it is hypothesized that niacin and thiamine supplementation at concentrations sufficient to sustain a 24 hour full vasodilatation in the micro circulation, will obviate the malfunction in the microcirculation, stop tissue damage immediately, and allow nature to restore functionality to organs.

\section{Objective}

The objective was to test the hypothesis by preparing a supplement containing niacin and thiamine as the active ingredients and administering it to type 2 diabetes mellitus patients with symptoms of diabetes complications.

\section{Method}

\section{There are two parts}

a. Formulating the form of therapy and

b. Use of the therapy by type 2 diabetes mellitus patients showing symptoms of diabetes complications.

\section{The therapeutic form}

In previous studies of the effect of vitamin B niacin ${ }^{3}$ and thiamine ${ }^{4}$ on human diabetes mellitus patients, daily ingestion of tablets containing $1000 \mathrm{mg}$ of niacin, and $300 \mathrm{mg}$ of thiamine, respectively, were used and no adverse effects were reported. So in this study, a tablet containing: nicotinamide, $15.0 \mathrm{mg}$; thiamine hydrochloride, $12.5 \mathrm{mg}$; and calcium-d-pantothenate (b5), $11.0 \mathrm{mg}$; to be ingested one a day, was used. Vitamin b5 was added for its ability to: accelerate the healing of skin wounds; ${ }^{5}$ relieve muscle cramps; ${ }^{6}$ cure numbness in fingers and toes and the painful 'burning feet syndrome'; ${ }^{7}$ and aid sperm generation. ${ }^{8}$ (The Low Dose Vitamin B Therapy (LDVbT) preparation, packaged 60 tablets/tub, was named "fenomin vitamin supplement" to differentiate it from commercially available "vitamin B complexes").

Dosage: One fenomin tablet is ingested daily by the patient in the evening after meals.

\section{Open trial}

The open trial of LDVbT started in 2008 with the author and expanded to include others 12 months later. Included were persons aged 60years or over with type 2 diabetes mellitus and manifesting 
one or more symptoms of diabetes complications. They would be using only tablets to control their blood glucose level.

Participants were advised that fenomin treats or prevents diabetes complications only, not diabetes mellitus, so they should continue using their prescription medications for diabetes mellitus during the trial. Advice on diet and exercise was reiterated. No advice was given on what biochemical endpoint criteria to look for to support the cure or/and improvement observed. Healed sore, improved erectile function, warmed-up hands and feet; improved visual acuity, etc. did not require biochemical endpoint criteria to support it. Record of blood glucose levels and $\mathrm{HbA} 1 \mathrm{c}$ readings was not considered a requirement. The participants were encouraged to keep using the tablet even after their symptoms disappeared. Monitoring for vitamin toxicity was deemed unnecessary at the concentration level used.

The model does not recommend an "end" to the use of the therapy. However, some persons stopped using the tablets when the symptom for which they sought remedy, such as healed sore, but restarted using it after periods ranging from about 6 to 12 months. Most have continued to use the therapy as prophylactic even, as their symptoms disappeared. The results (patient's observations), were voluntarily communicated to the author by some of those who had used the therapy to treat complications of all kinds. He, then, filtered out those who did not meet the inclusion criteria. No adverse effect has been reported to date. Table1 shows symptom of diabetes complication cured or improved and the number of patients reporting the cure or improvement, as at 31/08/2014.

Table I Symptoms of diabetes complication and number reported

\begin{tabular}{ll}
\hline Complication & No. Reported \\
\hline Leg ulcers healed & 16 \\
Scalp ulcers healed & 1 \\
Foot/Toe ulcers healed & 25 \\
Cold hands and feet warmed up & 18 \\
Improved visual acuity & 21 \\
Sore gums healed & 3 \\
Erectile function restored/improved & 34 \\
Swollen feet healed & 12 \\
Severe itching in genital area stopped & 14 \\
Semen production restored & 14 \\
Numbness in toes and fingers disappeared & 18 \\
Lowered, stable blood glucose level & 19 \\
Lowered cholesterol level & 19 \\
"Burning feet syndrome" stopped & 23
\end{tabular}

\section{Discussion}

The results have validated the major conclusions arising from the model. Some persons reported cure or improvement of symptoms manifesting in more than one organ. Four persons, who already had stroke and later developed leg-sore, took fenomin. They reported healed sore as well as improved quality of life. People reporting "decreased cholesterol and lowered blood plasma" levels were obviously reporting from their medical records. These improvements may have come about as part of the "feel-good" factor expressing itself in healthy eating and increased mobility such as, walking faster and further.

Because the trial is continuing, the list in the table is merely indicative of the width and scope of diabetes complications cured or improved. Reported improvement in the quality of live, such as becoming more active and feeling healthier, could not be quantified. (The trial is taking place in the UK and participants' health records are available in their doctors' surgeries, but were not accessible to this report). Two people who halted the therapy for some six months were hospitalized for complications before coming back on the therapy. They reported to be well.

The reported, ${ }^{9,10}$ beneficial effects of High Dose Benfotiamine/ Thiamine Therapy $(\mathrm{HDB} / \mathrm{T})$ on dysfunctional kidney, retinopathy, and "other benefits" can be explained by the blood plasma Vitamin B Deficiency theory. In both molecules of the lipid-soluble benfotiamine and water-soluble thiamine, the methylene link cleaves ${ }^{11}$ to give the water-soluble pyrimidinium moiety. The Pyrimidine molecule is chemically similar to the pyrine of niacin and acts as a vasodilator in the microcirculation. The high dose ensures 24 hour full vasodilatation in the microcirculation to enable cure or improvement to take place.

\section{Comparison of the glucose model with the vitamin B deficiency model}

The glycation theory of the glucose model posits that all organs are at equal risk of developing diabetes complications. This does not reflect observed facts. It necessitates the postulate of an active intermediate, Thiamine pyrophosphate (TPP), to stop glycation that produces (AGES), but does not explain how the "bad" particles already causing organ dysfunction are removed to effect cure or improvement. By stopping glycation, blood glucose level is expected to go up and $\mathrm{HbAlc}$ down during treatment but this is not observed, indicating that the improvements are independent of hyperglycemia.

High dose treatment of both thiamine and benfotiamine are not user-friendly for daily long-term use. The 12 week study and the 8 week run off time allowed in HDBT open trials are in adequate in evaluating efficacy. There is no indication of what happens to the cure or improvement before and after treatment in the longterm. The Vitamin B Deficiency (VbD) model predicts correctly that organs with microcirculations are at higher risk of developing diabetes complications. It explains clearly how complications develop simultaneously in these organs, predicts the appropriate therapy and consequence of treatment withdrawal. The model prediction of $100 \%$ efficacy has been demonstrated in the open trial.

\section{Conclusion}

The cures, improvements and prevention of diabetes complications accomplished by the therapy predicted by the Blood Plasma Vitamin B Deficiency model makes the model a potent tool in the study of diabetes complications.

\section{Perspective}

The vitamin B supplementation therapy, derived from the VbD model, has been demonstrated as on stop, efficacious treatment for all manifestations of diabetes complications in all organs for over five years. It is user-friendly, cheap, does not interact with other drugs and has no side effects. The treatment does not require medical supervision: patients take fenomin tablets alongside their prescription drugs. The cure for diabetes complications by the LDVbT is a major breakthrough in the fight against the predicted type 2 diabetes mellitus 
epidemic. ${ }^{12}$ It means that in the future, diabetes mellitus patients need not live in fear of amputation of limbs, blindness, ischemic stroke and heart attack or kidney failure. The social and economic impact of preventing diabetes complications by using fenomin tablets as prophylactic is immense.

\section{Recommendations}

More data are needed to confirm the findings. But a third stage, randomized, double-blind, placebo-controlled clinical trial for, maybe, ten to fifteen years, may not be the correct approach because the conditions for such a study do not apply here.

In a typical clinical trial, the test subjects "complete" the treatment before assessment can be made. In the LDVbT, the healing and prevention are continuing processes and the results are their own proof. The day the therapy is stopped is the day tissue damage restarts. However, fenomin can be used to heal sores instead of amputation; clear blurred vision to stop blindness and heal swollen feet to stop kidney failure, now. This work has excluded persons with type 1 diabetes mellitus for obvious reasons, so a separate supervised study must be conducted.

\section{Acknowledgements}

i. This research received no funding from public, private or notfor-profit Organizations.

ii. This article is published under open access Creative Commons Attrubution-NonCommercial ShareAlike (CCBY-NC-SA4.0")

\section{Conflict of interest}

Author declares that there is no conflict of interest.

\section{References}

1. Rabbani N, Thornalley PJ. Emerging role of thiamine therapy for prevention and treatment of early stage diabetic nephropathy. Diabetes Obes Metab. 2011;13(7):577-583.
2. Thornalley PJ, Babaei-Jadidi R, Al Ali H, et al. High prevalence of low plasma thiamine concentration in diabetes linked to a marker of vascular disease. Diabetologia. 2007;50(10):2164-2170.

3. Gale EA. Theory and practice of Nicotinamide trials in pre-Type 1 diabetes. J Pediatr Endochrinol Metab. 1996;9(3):375-379.

4. Rabbani N, Alam SS, Riaz S, et al. High dose thiamine therapy for patients with type2 diabetes and microalbuminurea; a pilotrandomised, doubleblind placebo controlled study. Diabetologia. 2009;52(2):208-212.

5. Weimann BJ, Hermann D. Studies in wound healing: Effect of Calcium D-pantothenate on the migration, proliferation and protein synthesis of human dermal fibroblasts in culture. Int $J$ Vitam Nutr Res. 1999;69(2):113-119.

6. Medeiros DM. Dietary reference intakes: The essential guide to nutrient requirements. In: Otten JJ, editor. Washington, DC, USA: The National Academies Press; 2006. p. 560.

7. Hodges RE, Ohlson MA, Bean WB. Pantothenic acid deficiency in man. J Clin Invest. 1958;37(11):1642-1657.

8. Kuo YM, Hayflick SJ, Gitschier JJ. Deprivation of pantothenic acid elicits a movement disorder and azoospermia in a mouse model of pantothenate kinase-associated neurodegeneration. J Inherit Metab Dis. 2007;30(3):310-317.

9. Hammes HP, Du X, Edelstein D, et al. Benfotiamine blocks three major pathways of hyperglycaemic damage and prevents experimental diabetic retinopathy. Nat Med. 2003;9(3):294-299.

10. Babaei-Jadidi R, Karachalias N, Ahmed N, et al. Prevention of incipient diabetic nephropathy by high dose thiamine and Benfotiamine. Diabetes. 2003;52(8):2110-2120.

11. Ziporin ZZ, Nunes WT, Powell RC, et al. Thiamine requirement in the adult human as measured by urinary excretion of thiamine metabolites. $J$ Nutr. 1965;85:297-304.

12. Chen L, Magliano DJ, Zimmet PZ. The worldwide epidermiology of type 2 diabetes mellitus - present and future perspectives. Nat Rev Endocrinol. 2011;8(4):228-236. 